\title{
EL TRATAMIENTO INFORMATIVO DE LAS ELECCIONES DE 2009 A LA EUROCÁMARA: COMICIOS DE SEGUNDO ORDEN Y EN CLAVE NACIONAL
}

\author{
Patricia Lafuente Pérez \\ (Universidad San Jorge) \\ alafuente@usj.es \\ Cristina Zurutuza \\ (Universidad San Jorge) \\ czurutuza@usj.es
}

\begin{abstract}
Resumen: El 7 de junio de 2009 se celebraron las elecciones al Parlamento Europeo en España. La campaña que los partidos desplegaron y la cobertura informativa que los medios hicieron de ésta, suscita la cuestión de si estos comicios despertaron interés por sí mismos. Esta investigación, mediante un análisis de contenido cuantitativo aplicado a los diarios El País, EI Mundo, Heraldo de Aragón y El Periódico de Aragón, pretende delimitar la relevancia de dicha campaña en la agenda mediática e identificar a sus protagonistas, así como la tematización dominante para comprobar si fueron abordados en clave europea, nacional o autonómica.
\end{abstract}

Palabras Clave: cobertura informativa, comicios, Parlamento Europeo, prensa nacional, prensa regional.

Abstract: Last European Parliament Elections were held the 7th of June, 2009, in Spain. The campaign that political parties displayed, and its media coverage raise questions about if this electoral contend awoken interest by itself. This research, through a quantitative content analysis applied to the newspapers El País, El Mundo, Heraldo de Aragón y El Periódico de Aragón, aims to set the relevance of the European Parliament campaign in the media agenda, and to identify the predominant issues and political characters in order to determine if they were reported through a European, national or regional approach.

Keywords: elections, European Parliament, news coverage, regional press, Spanish press.

\section{INTRODUCCIÓN}

I proceso de integración europeo ha supuesto la cesión voluntaria de soberanía desde los parlamentos estatales hacia el Parlamento Europeo y ha provocado que un gran número de medidas que afectan a la vida diaria de los ciudadanos comunitarios se decida fuera de las fronteras de sus 
El tratamiento informativo de las elecciones de 2009 a la Eurocámara: comicios de segundo orden y en clave nacional

respectivos países. A pesar de este hecho, y a tenor de los datos sobre índices de participación, las urnas reflejan una tendencia a atribuir poca relevancia a las elecciones que eligen a los miembros de esta institución legislativa. Desde que se convocaron las primeras elecciones al Parlamento Europeo en 1979, las cifras indican una diferencia de unos 25 puntos porcentuales entre los comicios generales dentro de cada Estado y los europeos (Morata, 2000: 22; Steinbrecher y Huber, 2006).

España no es ajena a esta tendencia. Aunque el Parlamento Europeo tiene cada vez una mayor capacidad de decisión e influencia en asuntos que afectan internamente a nuestro país, los datos reflejan que el interés que despierta en los ciudadanos este tipo de comicios es claramente inferior a otras citas electorales. Sirva como ejemplo que el nivel de abstención en las europeas de 2009 alcanzó al 50,1\% del electorado, mientras que en las legislativas del año 2008 la participación fue del 73,85\%"

Las últimas elecciones europeas, celebradas en España el 7 de junio de 2009, no han sido una excepción en cuanto a la participación electoral y la lectura nacional de la campaña, como se explica en las siguientes páginas. Los comicios de junio, en los que al electorado español le correspondía elegir 50 de los 736 eurodiputados que componen actualmente el Parlamento Europeo, tuvieron una participación del $44,9 \%$. Algo más de la mitad de los ciudadanos con derecho a voto optaron por no ejercerlo, siendo las de 2009 las elecciones al Parlamento Europeo con una mayor tasa de abstencionismo desde que se celebraran por primera vez en España en 1987. De las 35 candidaturas presentadas, únicamente 6 consiguieron representación. Los escaños quedaron repartidos de la siguiente manera: 23 para el Partido Popular (PP), 21 para el Partido Socialista Obrero Español (PSOE), 2 para Coalición por Europa (CEU), 2 para la coalición formada por Izquierda Unida, Iniciativa por Catalunya-Verds, Esquerra Unida i Alternativa y Bloque por Asturies: la Izquierda (IU-ICV-EUIABA), 1 para Unión, Progreso y Democracia (UPyD) y 1 para Europa de los Pueblos-Verdes (EDP-V) ${ }^{2}$.

Esta distribución de escaños es también un indicativo claro del planteamiento de corte nacional que tanto partidos como ciudadanos hicieron de la campaña electoral al Parlamento Europeo. Se trata de unos comicios para la elección de un órgano legislativo supranacional para los que España funciona como una circunscripción única, a diferencia de las elecciones generales, en las que cada provincia se constituye en circunscripción. Es decir, el escenario que a priori dibuja la legislación es completamente diferente al de cualquier otro proceso interno. Sin embargo, la aplicación de la Ley D'Hondt como sistema

${ }^{1}$ Datos del Ministerio del Interior (www.mir.es). ¿'́dem. 
de reparto proporcional corregido de escaños, al igual que en las generales, y el tipo de comportamiento de voto del ciudadano, reflejado en los resultados, dan lugar a un peculiar sistema de partidos circunscrito al ámbito nacional pero proyectado hacia Europa, caracterizado por una tendencia a asimilar el patrón de conducta de las elecciones a Cortes Generales. El multipartidismo moderado, con una tendencia al bipartidismo cada vez más acusada, que caracteriza la configuración del Parlamento español, vuelve a darse en las elecciones al Parlamento Europeo: PP y PSOE consiguen la mayor parte de los escaños, muy por encima de los siguientes partidos con representación.

Los niveles de participación habitualmente bajos pueden deberse a las dificultades para identificar y entender con claridad a esta institución europea. Como señalan Canel e Innerarity "el sentimiento de competencia electoral desciende" (2000: 171) debido a la magnitud de estos comicios. Los obstáculos del ciudadano europeo para conocer y controlar el trabajo de sus representantes europeos tienen un carácter disuasorio para el electorado en cuanto a su implicación en los procesos de constitución y renovación del organismo supranacional (Ruiz, 2004; García y Pérez, 2009). Esto provoca, además, que crezca la percepción del Parlamento Europeo "como una institución poco visible, alejada y anómala que no elige un ejecutivo ni legisla" (Morata, 2000: 22).

Otra de las causas de este desinterés reside en el planteamiento que los propios partidos políticos hacen de su campaña para estos comicios, pues ven en ella una oportunidad para "medir sus niveles de popularidad" y testar su situación política interna (Berganza, 2008a: 17). La inexistencia de un sistema de partidos europeo y de un único sistema electoral y, por tanto, de "una verdadera campaña europea" (Morata, 2000: 23) influye de manera decisiva en este aspecto, restringiendo en cierto modo la proyección de los partidos, por ende, de sus temas y candidatos, y confinando sus campañas a un ámbito de actuación similar al de otras citas electorales de orden interno.

Sin embargo, esta consideración secundaria de las elecciones europeas por parte de los diferentes actores políticos implicados no se explica sólo por los altos niveles de abstención, ni es debido únicamente al diseño de la campaña por los partidos. Un tercer factor es el tipo de cobertura que llevan a cabo los medios.

\section{ESTADO DE LA CUESTIÓN}

Numerosos expertos han señalado que los medios nacionales cubren las elecciones europeas como comicios de segundo orden (Canel e Innerarity, 2000: 171; Reif, 1985; Maier y Tenscher, 2006; De Vreese, 2006 y 2009; Maarek, 2007; Berganza, 2008b: 127; Tenscher y Maier, 2009; Tigasson, 2009). Esto explica que predominen los temas de política interna y los enfoques o encuadres nacionales frente a los comunitarios. Lo prioritario no es mostrar el debate sobre cuestiones 
El tratamiento informativo de las elecciones de 2009 a la Eurocámara: comicios de segundo orden y en clave nacional

relacionadas directamente con Europa.

No obstante, esta tendencia a nacionalizar los temas por parte de los medios, explica Berganza, es una constante que caracteriza la información comunitaria más allá de los procesos electorales. La descripción y valoración desde una perspectiva estatal de los temas que afectan a Europa "es comprensible y resulta pertinente" (2005: 139). Si el sentido último del proceso de integración europea es, precisamente, la defensa de los intereses de los ciudadanos, entonces "lógicamente los mensajes provenientes de Europa tendrán que apelar constantemente a la salvaguarda de los intereses nacionales que supone la propia presencia en Europa" (Dahl, 1999: 134, citado en Canel e Innerarity, 2000: 172; Sotelo, 2008). Las informaciones, por tanto, tendrán que explicar qué repercusiones tienen las decisiones de la UE en cada país, traduciendo en términos nacionales lo que suceda en Europa. Sin embargo, se echa en falta una visión de conjunto que ayude a la ciudadanía a valorar de manera acertada algunas cuestiones y a comprender de forma más precisa lo que es la realidad comunitaria (Berganza, 2005: 139).

Anteriores estudios han evidenciado, además, que en la cobertura de las elecciones europeas hay un elevado grado de personalización. Como es habitual en otras campañas electorales, "el mensaje electoral está representado en una persona concreta" (Canel, 2006: 48) que funciona como gancho y con la que el votante puede identificarse más fácilmente. Sin embargo, en el caso de los comicios europeos esa propuesta electoral no se encarna en el candidato al Parlamento Europeo. Dado que los partidos consideran esta campaña como una especie de ensayo para medir sus fuerzas de cara a las elecciones generales, los líderes de esos partidos suelen tener una elevada visibilidad en los medios y eclipsan a los propios candidatos europeos (Berganza, 2008a: 27).

\section{OBJETIVOS Y METODOLOGÍA}

El primer objetivo de este trabajo es describir cómo fue la cobertura de las elecciones europeas de 2009 y dirimir si suscitaron interés por sí mismas o si, una vez más, mantuvieron su carácter secundario y fueron tratadas por los medios de comunicación en clave estatal.

El segundo es conocer si ese nivel de localización de la información afectó también, y de qué manera lo hizo, a los medios regionales. De esta manera, esta investigación da un paso más allá e introduce otro elemento al debate con el fin de contribuir a delimitar aún más ese tendente carácter "etnocentrista" (Berganza, 2008a: 25) de las elecciones europeas. Hasta ahora se ha demostrado que los medios de difusión nacional otorgan menos relevancia y un enfoque estatal a los comicios al Parlamento Europeo, en este estudio se pretende concretar hasta qué nivel se traduce la cuestión europea: si esa lectura se circunscribe únicamente al ámbito nacional o, además, desciende hasta el nivel regional y autonómico. 
Para la consecución de estos dos objetivos se toma como ejemplo el caso de la cobertura realizada en la Comunidad de Aragón. Se analizan los dos principales medios de tirada nacional, El País y El Mundo, y en el ámbito autonómico aragonés sus dos cabeceras de referencia: Heraldo de Aragón y El Periódico de Aragón ${ }^{3}$.

Las cifras de difusión de los citados medios justifican esta elección: El País (431.033 ejemplares) y El Mundo (323.587 unidades) son los periódicos de información general de mayor difusión en España (AEDE, 2010: 46-47). En cuanto a los diarios aragoneses, Heraldo de Aragón, editado en Zaragoza, es la cabecera más importante de la región, con una difusión media de 53.087 ejemplares en 2008; muy por encima de su principal competidor, El Periódico de Aragón (12.087 ejemplares). Trasladados estos datos de difusión al mercado aragonés se observa que Heraldo de Aragón domina la prensa diaria de esta región del nordeste peninsular pues supone el $52,3 \%$ del total mientras que El Periódico de Aragón representa el $12,5 \%$. Por detrás, se encuentran El País $(10,4 \%$ y El Mundo (6,1\%) (AEDE, 2010: 83). Se trata, por tanto, de los diarios nacionales con mayor difusión en Aragón así como de las dos cabeceras regionales de mayor peso en esta Comunidad española.

El método de investigación utilizado es el análisis de contenido cuantitativo, una técnica que permite, mediante la "aplicación sistemática de unas reglas fijadas previamente", medir la frecuencia de aparición de unos elementos relevantes dentro del conjunto estudiado, útiles para alcanzar los objetivos de la investigación (Sánchez Aranda, 2005: 213).

Se analizaron todas las piezas que contienen referencias a los comicios europeos durante las dos semanas que duró la campaña electoral previa a la elección del Parlamento Europeo (desde el 23 de mayo, día de inicio de la campaña, hasta el domingo 7 de junio, la jornada electoral) en los cuatro rotativos. El diseño del código se centra en aspectos de interés como la extensión de las informaciones, la sección en la que se ubican, los principales temas tratados, el enfoque otorgado a éstos $y$, por último, los protagonistas con más peso.

En total, se analizaron 866 informaciones: 252 correspondientes a El País, 260 a El Mundo, 201 a Heraldo de Aragón y 153 a El Periódico de Aragón. Si se compara el volumen de noticias publicadas por estos periódicos con el número de informaciones de otras campañas electorales se evidencia ya el carácter secundario otorgado a estos comicios europeos de 2009. Así, en las elecciones generales de 2008, durante las dos semanas de la campaña, entre estos mismos periódicos se publicaron 1925 unidades distribuidas del siguiente modo: 381 en El País, 550 en El Mundo, 522 en Heraldo de Aragón y 472 en El Periódico de Aragón (Zugasti, et al., 2011: 17).

\footnotetext{
${ }^{3}$ Este trabajo forma parte de los resultados del grupo de investigación "Medios de comunicación y campañas electorales en Aragón", reconocido como grupo de investigación emergente por el Gobierno de Aragón.
} 
El tratamiento informativo de las elecciones de 2009 a la Eurocámara:

comicios de segundo orden $y$ en clave nacional

\section{HIPÓTESIS DEL ESTUDIO}

Este trabajo pretende confirmar o refutar, por un lado, que las elecciones de junio de 2009 mantuvieron su carácter secundario y fueron tratados por los medios de comunicación en clave estatal. Es decir, conocer si este proceso electoral supranacional continúa teniendo un carácter nacional.

La segunda hipótesis de partida de esta investigación es que, frente a una posible europeización del discurso informativo, los medios de ámbito autonómico ofrecen un enfoque regional.

\section{ANÁLISIS DE LOS DATOS}

Desde el 23 de mayo, fecha de inicio de la campaña electoral, hasta el 7 de junio de 2009, jornada en la que los españoles debían depositar su voto en las urnas, los cuatro diarios observados generaron 866 unidades informativas sobre las elecciones al Parlamento Europeo. A continuación se describen los principales rasgos de la cobertura en los cuatro rotativos.

\subsection{Rasgos formales}

Si se considera que el número de noticias sobre la campaña electoral está directamente relacionado con la importancia que cada periódico le dio a esta cuestión, se puede avanzar la siguiente conclusión: la relevancia dada a las elecciones no fue idéntica en los cuatro casos, pues al comparar la acumulación de noticias se observan diferencias (tabla 1); sin embargo, las disparidades sobre este aspecto fueron mínimas.

Tabla 1. Número de noticias por diario

\begin{tabular}{|l|c|c|}
\hline Periódico & Noticias & Porcentaje \\
\hline El País & 252 & 29,09 \\
\hline El Mundo & 260 & 30,03 \\
\hline El Heraldo de Aragón & 201 & 23,22 \\
\hline El Periódico de Aragón & 153 & 17,66 \\
\hline Total & 866 & 100 \\
\hline
\end{tabular}

Fuente: Elaboración propia. 
Junto al número de piezas, algunos rasgos formales en la presentación de las noticias, como la extensión y la ubicación del texto, son reveladores del grado de interés que un medio de comunicación concede a una cuestión. En este caso, en cuanto a la extensión, la mayoría de las noticias, más del $76 \%$ de las piezas, fueron medianas y cortas. Por tanto, los cuatro diarios descartaron otorgar una posición más destacada a las informaciones sobre estos comicios utilizando piezas de mayor extensión.

Tabla 2. Extensión de las noticias en los cuatro diarios

\begin{tabular}{|l|c|}
\hline \multicolumn{1}{|c|}{ Extensión } & Porcentaje \\
\hline Muy larga & 7,97 \\
\hline Larga & 15,94 \\
\hline Mediana & 37,29 \\
\hline Corta & 38,80 \\
\hline Total & 100 \\
\hline
\end{tabular}

Fuente: Elaboración propia.

De nuevo, de igual modo que en el número de informaciones publicadas por cada medio, las diferencias entre los diarios fueron mínimas, independientemente de que se esté ante una cabecera nacional o regional.

Tabla 3. Extensión de las noticias por diario

\begin{tabular}{|l|c|c|c|c|}
\hline Extensión & El País & El Mundo & Heraldo de Aragón & $\begin{array}{c}\text { El Periódico de } \\
\text { Aragón }\end{array}$ \\
\hline Muy larga & 3,99 & 13,07 & 3 & 12,44 \\
\hline Larga & 15,07 & 15,7 & 14,52 & 19,6 \\
\hline Mediana & 34,12 & 36,15 & 35,52 & 46,4 \\
\hline Corta & 46,82 & 35,08 & 46,96 & 21,56 \\
\hline Total & 100 & 100 & 100 & 100 \\
\hline
\end{tabular}

Fuente: Elaboración propia.

Además de la extensión, el lugar donde se presenta la noticia es una de las formas de valorar su importancia respecto al resto de las piezas publicadas en un diario. En el caso de la prensa escrita la portada muestra los temas más relevantes para el medio. Durante la campaña electoral de 2009 este recurso fue escasamente utilizado en los cuatro medios, pues sólo 47 piezas sobre los comicios fueron publicadas en este espacio, lo que supone únicamente un 5,4\% de todas las unidades analizadas. Es destacable también que, a pesar de tratarse de unas elecciones de corte europeo, por tanto, trasnacionales, apenas se ubicó este tema en Internacional. 
70 El tratamiento informativo de las elecciones de 2009 a la Eurocámara: comicios de segundo orden y en clave nacional

De hecho, sólo se incluyeron en esta sección 87 piezas de las 866 publicadas. De los medios observados. El Mundo fue el que más ubicó las informaciones en este apartado. Por el contrario, en la mayor parte de las ocasiones (cerca del $50 \%$ ) las noticias fueron publicadas dentro de la sección nacional. En este sentido, no es posible establecer grandes diferencias entre los diarios observados, pues incluso los dos diarios regionales optaron por incluir más informaciones en Nacional que en la sección dedicada exclusivamente a noticias sobre Aragón (véase tabla 5).

Las piezas de análisis también tuvieron su espacio durante la cobertura electoral: en total, 156 unidades fueron ubicadas en la sección de Opinión. Los medios regionales publicaron más informaciones en estas páginas dedicadas a la argumentación y las valoraciones. También, como era de esperar por la naturaleza de dos de los medios que forman parte de nuestra muestra, se codificaron 114 informaciones en Regional. En cuanto a la ubicación de las noticias cabe destacar finalmente que, a pesar de que tanto Heraldo de Aragón como El Periódico de Aragón tienen secciones locales, ninguna información sobre las elecciones se publicó en estos espacios.

Tabla 4. Ubicación en los cuatro diarios

\begin{tabular}{|l|c|}
\hline \multicolumn{1}{|c|}{ Ubicación } & Porcentaje \\
\hline Nacional & 49,66 \\
\hline Opinión & 18.01 \\
\hline Regional & 13,17 \\
\hline Internacional & 10,06 \\
\hline Portada & 5,42 \\
\hline Contraportada & 1,15 \\
\hline Sociedad & 0,8 \\
\hline Economía & 0,34 \\
\hline Otros & 1,38 \\
\hline Total & 100 \\
\hline
\end{tabular}

Fuente: Elaboración propia.

Los diarios El País, Heraldo de Aragón y El Mundo fueron, por este orden, los que más utilizaron la portada para informar sobre las inminentes elecciones, como se puede observar en la tabla 5. En el segundo de los medios regionales, El Periódico de Aragón, sólo se utilizó este recurso en cuatro ocasiones. De modo que es imposible establecer un único patrón sobre este aspecto para caracterizar a los medios nacionales frente a los regionales. Los medios aragoneses sí coinciden al publicar menos piezas en la sección nacional que los dos rotativos con sede en Madrid. 
Tabla 5. Ubicación por diarios (porcentaje).

\begin{tabular}{|l|c|c|c|c|}
\hline Ubicación & El País & El Mundo & Heraldo de Aragón & $\begin{array}{c}\text { El Periódico de } \\
\text { Aragón }\end{array}$ \\
\hline Portada & 7,20 & 5,38 & 6,40 & 2,71 \\
\hline Contraportada & 1,98 & 1,15 & 0,99 & 0 \\
\hline Opinión & 11,71 & 19,24 & 19,40 & 27,24 \\
\hline Economía & 0,79 & 0,38 & 0 & 0 \\
\hline Nacional & 65,07 & 52,69 & 40,59 & 31,81 \\
\hline Internacional & 9,88 & 20,77 & 3,98 & 0 \\
\hline Regional & 0 & 0 & 28,15 & 31,06 \\
\hline Otros & 3,37 & 0,39 & 0,49 & 7,18 \\
\hline Total & 100 & 100 & 100 & 100 \\
\hline
\end{tabular}

Fuente: Elaboración propia.

\subsection{Temas y protagonistas nacionales para una campaña europea}

Algunos estudios han puesto de evidencia que durante las campañas electorales es "difícil diferenciar entre los mensajes europeos, municipales y autonómicos en la cobertura informativa" (Berganza, 2008a: 25) puesto que los propios partidos políticos apenas distinguen entre propuestas sobre política nacional y europea. Coincidiendo con esta afirmación, en la cobertura de 2009 los temas de campaña que más importancia tuvieron (los que al menos focalizaron las informaciones en un $4 \%$ de las ocasiones) fueron, por este orden, los siguientes: corrupción política, participación en las elecciones y euroescepticimismo, crisis económica, instituciones de la Unión Europea, otros asuntos económicos, organización interna de los partidos políticos para las elecciones, legalización de los brazos políticos de la banda terrorista ETA, políticas de descentralización territorial y debate del Estado autonómico.

Como se puede deducir de los temas enumerados más arriba, sólo dos cuestiones están relacionadas con política comunitaria, mientras que el resto se refieren a aspectos de la esfera nacional que estaban presentes en la opinión pública española antes del comienzo de la campaña electoral. Además, el tema de corte europeo que más importancia tuvo en la cobertura (participación/abstención/ euroescepticismo) implicaba en la mayoría de las ocasiones una visión negativa o, al menos pesimista, de la relación entre el electorado español con las instituciones europeas. 
72 El tratamiento informativo de las elecciones de 2009 a la Eurocámara: comicios de segundo orden y en clave nacional

Tabla 6. Principales temas en los cuatro diarios.

\begin{tabular}{|l|l|}
\hline Tema & Porcentaje \\
\hline Corrupción /escándalos políticos & 17,55 \\
\hline Participación/abstención en las elecciones/euroescepticismo & 11,66 \\
\hline Crisis económica & 9,93 \\
\hline Instituciones de la UE & 7,97 \\
\hline Otros asuntos económicos & 5,19 \\
\hline Organización de los partidos políticos para las elecciones & 4,97 \\
\hline Legalización/ilegalización de brazos políticos de ETA & 4,85 \\
\hline Descentralización territorial/Estado autonómico & 4,85 \\
\hline Datos de encuestas relativas a las elecciones & 2,88 \\
\hline Infraestructuras & 2,54 \\
\hline Aborto & 1,84 \\
\hline Educación & 1,5 \\
\hline Referencias al pasado político español & 1,38 \\
\hline Inmigración & 1,38 \\
\hline $\begin{array}{l}\text { Relaciones con países miembros de la UE } \\
\text { y/o con instituciones de la UE }\end{array}$ & 1,15 \\
\hline Medio ambiente & 0,8 \\
\hline Sanidad & 0.8 \\
\hline Relaciones exteriores con países no miembros de la UE & 0,6 \\
\hline Energía & 0,44 \\
\hline Otros & 17,72 \\
\hline Total & 100 \\
\hline
\end{tabular}

Fuente: Elaboración propia.

Tal y como se puede observar en la tabla 7 apenas hay diferencias entre los principales temas de la cobertura informativa en cada uno de los diarios. En todos ellos las diferentes cuestiones de política nacional tuvieron bastante más peso que las comunitarias. En los diarios El País, El Mundo y Heraldo de Aragón, los casos de corrupción política coparon casi toda la atención; un hecho que se explica porque el caso Gürtel, vinculado al Partido Popular, fue conocido por la opinión pública unos meses antes al comienzo de la campaña. Además, durante el intervalo del 23 de mayo al 5 de junio el uso de un avión Falcon por el presidente del Gobierno español, José Luis Rodríguez Zapatero, para acudir a actos de su partido fue uno de los argumentos más frecuentes en el cruce de acusaciones entre los grandes partidos políticos del país.

El caso de El Periódico de Aragón es particular en este sentido, pues se 
desmarca de este patrón ya que el tema principal durante su cobertura fue la abstención electoral y el euroescepticismo. Llama la atención también que los dos diarios regionales recojan menos informaciones sobre el Estado autonómico y la descentralización regional que los dos rotativos nacionales.

Tabla 7. Principales temas en cada diario (porcentaje).

\begin{tabular}{|l|c|c|c|c|}
\hline Tema & El País & El Mundo & $\begin{array}{c}\text { Heraldo de } \\
\text { Aragón }\end{array}$ & $\begin{array}{c}\text { El Periódico } \\
\text { de Aragón }\end{array}$ \\
\hline Corrupción /escándalos políticos & 22,34 & 15,38 & 17,91 & 14,37 \\
\hline $\begin{array}{l}\text { Participación/abstención en las } \\
\text { elecciones/euroescepticismo }\end{array}$ & 8,9 & 10,38 & 14,92 & 15,68 \\
\hline Crisis económica & 10,27 & 8,84 & 7,46 & 14,37 \\
\hline Instituciones de la UE & 8,3 & 12,43 & 9,95 & 1,96 \\
\hline Otros asuntos económicos & 5,92 & 1,53 & 7,96 & 6,53 \\
\hline $\begin{array}{l}\text { Organización de los partidos } \\
\text { políticos para las elecciones }\end{array}$ & 4,74 & 6,72 & 1,99 & 7,18 \\
\hline $\begin{array}{l}\text { Legalización/ilegalización de } \\
\text { brazos políticos de ETA }\end{array}$ & 5,92 & 6,15 & 2,48 & 3,92 \\
\hline $\begin{array}{l}\text { Descentralización territorial/Es- } \\
\text { tado autonómico }\end{array}$ & 3,95 & 8,46 & 2,48 & 3,26 \\
\hline $\begin{array}{l}\text { Datos de encuestas relativas a } \\
\text { las elecciones }\end{array}$ & 2,76 & 4,61 & 0,99 & 2,61 \\
\hline Infraestructuras & 1,58 & 0,76 & 5,97 & 2,61 \\
\hline Aborto & 2,76 & 1,53 & 0,99 & 2,61 \\
\hline Educación & 2,37 & 0 & 1,49 & 2,61 \\
\hline $\begin{array}{l}\text { Referencias al pasado político } \\
\text { español }\end{array}$ & 1,97 & 1,15 & 0,49 & 1,96 \\
\hline Inmigración & 0,39 & 2,69 & 0,49 & 1,96 \\
\hline $\begin{array}{l}\text { Relaciones con países miem- } \\
\text { bros de la UE } \\
\text { y/o con instituciones de la UE }\end{array}$ & 0,39 & 0 & 2,98 & 1,96 \\
\hline Medio ambiente & 0,39 & 1,33 & 0,99 & 1,3 \\
\hline Sanidad & 1,18 & 1,33 & 0 & 2,3 \\
\hline $\begin{array}{l}\text { Relaciones exteriores con paí- } \\
\text { ses no miembros de la UE }\end{array}$ & 0,69 & 1,92 & 0 & 0 \\
\hline Energía & 1,15 & 0,38 & 0 & 0 \\
\hline Otros & 15 & 14,42 & 21,92 & 9,18 \\
\hline
\end{tabular}

Fuente: Elaboración propia. 
74 El tratamiento informativo de las elecciones de 2009 a la Eurocámara: comicios de segundo orden y en clave nacional

Por el contrario, la cuestión de las mejoras de las infraestructuras tuvo más peso en los dos periódicos aragoneses, sobre todo en Heraldo de Aragón, que en los nacionales. Un hecho que se explica por el debate en torno a la Travesía Central Pirenaica que afecta directamente a la Comunidad de Aragón y que es un tema constante en la agenda de los medios de esta región, independientemente de la existencia de una campaña electoral.

En cuanto al enfoque también se observó que el punto de vista nacional tuvo bastante más peso que el europeo en los cuatro diarios. Como se puede ver en la tabla 8 , en la cobertura se hicieron sobre todo referencias explícitas a la situación interna de España que nada o poco tenían que ver con lo que se decidía en los comicios del 7 de junio.

Tabla 8. Enfoque del tema principal en los cuatro diarios.

\begin{tabular}{|l|c|}
\hline \multicolumn{1}{|c|}{ Enfoque } & Porcentaje \\
\hline Nacional & 59,88 \\
\hline Europeo & 18,26 \\
\hline Nacional y europeo & 6,7 \\
\hline Otros & 15,16 \\
\hline Total & 100 \\
\hline
\end{tabular}

Fuente: Elaboración propia.

Como en el resto de los aspectos analizados en este trabajo apenas se pueden distinguir diferencias en el tipo de cobertura entre diarios. Incluso los dos medios de carácter regional optaron por tratar las noticias sobre la campaña electoral tomando como eje principal la situación en España y no la de Aragón.

Tabla 9. Enfoque del tema principal en cada diario (porcentaje).

\begin{tabular}{|l|c|c|c|c|}
\hline Enfoque & EI País & El Mundo & $\begin{array}{c}\text { Heraldo de } \\
\text { Aragón }\end{array}$ & $\begin{array}{c}\text { EI Periódico } \\
\text { de Aragón }\end{array}$ \\
\hline Nacional & 65,61 & 64,61 & 47,76 & 58,82 \\
\hline Europeo & 16,99 & 26,53 & 16,91 & 7,84 \\
\hline $\begin{array}{l}\text { Nacional y } \\
\text { europeo }\end{array}$ & 10,27 & 0 & 5,47 & 13,73 \\
\hline Aragonés & 0 & 0,38 & 13,93 & 15,68 \\
\hline $\begin{array}{l}\text { Aragonés y } \\
\text { europeo }\end{array}$ & 0 & 0 & 11,44 & 3,26 \\
\hline Otros & 7,13 & 8,48 & 4,49 & 0,67 \\
\hline Total & 100 & 100 & 100 & 100 \\
\hline
\end{tabular}

Fuente: Elaboración propia. 
Una situación similar se repite con los protagonistas de las informaciones. En seis protagonistas, de un total de 54 , se concentran el $41,13 \%$ de las informaciones. Además, frente a lo que podría parecer lógico por la naturaleza de estos comicios, en los cuatro medios los personajes principales no fueron los candidatos al Parlamento Europeo, sino el presidente del Gobierno, José Luis Rodríguez Zapatero, y el líder del PP, Mariano Rajoy. Probablemente siguiendo la estrategia electoral diseñada por los socialistas y los populares, estos comicios se trataron por los diarios como un enfrentamiento entre sus dos líderes, que fueron los protagonistas en aproximadamente un $11 \%$ de las ocasiones. En esta línea, los cabezas de lista Juan Fernando López Aguilar (PSOE) y Jaime Mayor Oreja (PP) fueron los personajes principales de las piezas en aproximadamente el $4 \%$ de las ocasiones, por debajo incluso de otros políticos nacionales que no concurrían a esas elecciones.

Tabla 10. Principales protagonistas en los cuatro diarios.

\begin{tabular}{|l|c|}
\hline \multicolumn{1}{|c|}{ Protagonistas } & Porcentaje \\
\hline $\begin{array}{l}\text { José Luis Rodríguez Zapa- } \\
\text { tero }\end{array}$ & 11,56 \\
\hline Mariano Rajoy & 10,98 \\
\hline $\begin{array}{l}\text { Otros políticos nacionales } \\
\text { del PSOE }\end{array}$ & 5,54 \\
\hline $\begin{array}{l}\text { Otros políticos nacionales } \\
\text { del PP }\end{array}$ & 5,08 \\
\hline $\begin{array}{l}\text { Juan Fernando López } \\
\text { Aguilar }\end{array}$ & 4,04 \\
\hline Jaime Mayor Oreja & 3,93 \\
\hline Otros (48 restantes) & 58,87 \\
\hline Total & 100 \\
\hline
\end{tabular}

Fuente: Elaboración propia.

Por otro lado, como se puede apreciar en la tabla 11, en los diarios regionales también hubo una concentración en sólo seis protagonistas.

Como los periódicos de Madrid, los aragoneses optaron por dar un lugar predominante a los líderes nacionales de los dos principales partidos de España. 
76 El tratamiento informativo de las elecciones de 2009 a la Eurocámara: comicios de segundo orden y en clave nacional

Tabla 11. Principales protagonistas por diarios (porcentaje).

\begin{tabular}{|l|c|c|c|c|}
\hline Protagonistas & EI País & El Mundo & $\begin{array}{c}\text { Heraldo de } \\
\text { Aragón }\end{array}$ & $\begin{array}{c}\text { EI Periódico } \\
\text { de Aragón }\end{array}$ \\
\hline $\begin{array}{l}\text { José Luis Rodrí- } \\
\text { guez Zapatero }\end{array}$ & 10,67 & 11,53 & 11,44 & 13,07 \\
\hline Mariano Rajoy & 9,88 & 11,53 & 8,45 & 15,03 \\
\hline $\begin{array}{l}\text { Otros políticos } \\
\text { nacionales del } \\
\text { PSOE }\end{array}$ & 5,92 & 6,71 & 4,47 & 4,57 \\
\hline $\begin{array}{l}\text { Otros políticos } \\
\text { nacionales del } \\
\text { PP }\end{array}$ & 5,53 & 4,61 & 4,97 & 5,22 \\
\hline $\begin{array}{l}\text { Juan Fernando } \\
\text { López Aguilar }\end{array}$ & 4,34 & 4,61 & 3,48 & 3,26 \\
\hline $\begin{array}{l}\text { Jaime Mayor } \\
\text { Oreja }\end{array}$ & 3,95 & 5,38 & 1,99 & 3,92 \\
\hline $\begin{array}{l}\text { Otros (48 restan- } \\
\text { tes) }\end{array}$ & 59,71 & 60,24 & 65,2 & 54,93 \\
\hline Total & 100 & 100 & 100 & 100 \\
\hline
\end{tabular}

Fuente: Elaboración propia.

En este sentido, sirva como ejemplo observar cómo los candidatos aragoneses por el PSOE o eurodiputados por el mismo partido sólo fueron protagonistas en 0,99\% de las ocasiones en Heraldo de Aragón y 1,3\% en El Periódico de Aragón. Una situación similar se repite con los candidatos aragoneses del PP: en Heraldo de Aragón protagonizaron $1,49 \%$ de las informaciones. Algo más elevada fue su presencia en El Periódico de Aragón, 2,6\%.

\section{CONCLUSIONES}

Tras haber comentado los resultados de esta investigación se observa que, independientemente de la adscripción geográfica de los rotativos estudiados, todos confieren un interés similar a la campaña de las elecciones al Parlamento Europeo de 2009 como hecho noticioso, pues el número de piezas que publica cada medio es parecido. Tampoco se destacan los comicios en las portadas de estos periódicos, lo cual muestra que el tema europeo no se encontraba entre las cuestiones más importantes de las jornadas analizadas. Desde el punto de vista formal se confirma que la magnitud de estos comicios fue secundaria. 
La lectura estatal de las elecciones comunitarias de 2009 en España, se comprueba, en primer lugar, en la sección donde se ubican las informaciones: más de la mitad aparecen en el área de Nacional, muy por encima de las que se incluyen en las páginas de Internacional o incluso en las regionales. Es necesario subrayar aquí que el ámbito geográfico de difusión de los rotativos no guarda una relación directa con la sección en la que se incluyen las informaciones. Tanto los periódicos nacionales como los regionales optan por ubicarlas principalmente en Nacional.

En segundo lugar, el encuadre nacional se observa también en los temas que más peso tienen en la cobertura electoral, ya que predomina el interés por cuestiones de corte interno por delante de asuntos de carácter europeo. La cobertura se convierte así en una continuación de los temas que ocupaban la agenda mediática antes del comienzo de la campaña. Este rasgo queda reforzado por el hecho de que el principal tema tratado que guarda conexión con Europa precisamente pone en cuestión la identificación de los ciudadanos españoles con el Parlamento Europeo y, por tanto, focaliza el debate en torno a los niveles de participación y el fantasma del abstencionismo. Tampoco en este aspecto puede afirmarse que exista una diferencia de comportamiento entre medios nacionales y regionales: todos otorgan mayor relevancia a temas circunscritos al ámbito estatal que a cuestiones europeas o aragonesas.

Además, el punto de vista nacional es también dominante a la hora de informar sobre los diferentes temas en todos los diarios, con un $60 \%$ de las 866 piezas analizadas tratadas desde una perspectiva estatal.

En tercer lugar, al igual que sucede con el enfoque, predomina la presencia de políticos nacionales que ni siquiera se presentaban como candidatos en las listas de cada partido. El protagonismo de José Luis Rodríguez Zapatero y Mariano Rajoy es un rasgo común a todos. Sin lugar a dudas son los candidatos propios de unas elecciones generales los que ocupan la mayor parte del espacio y del interés informativo, ya que aparecen muchas más veces que los cabezas de lista Juan Fernando López Aguilar y Jaime Mayor Oreja.

Estos resultados permiten concluir, por un lado, que no existe un patrón de comportamiento que permita distinguir un modo de informar sobre Europa característico de medios nacionales de otro propio de los regionales. El ámbito geográfico de difusión y, por tanto, el interés de los lectores de cada medio no determinan la adopción de un determinado enfoque en los periódicos. Cuando se trata de las elecciones europeas, todos los medios ofrecen un tipo de cobertura muy similar.

Por otro lado, esta investigación confirma lo que han demostrado estudios anteriores, es decir, que los comicios al Parlamento Europeo celebrados en España el 7 de junio de 2009 fueron un proceso electoral de segundo orden medido y traducido en términos nacionales. El encuadre estatal domina la cobertura de la campaña, sin diferencias destacables entre los diarios nacionales y regionales. 
78 El tratamiento informativo de las elecciones de 2009 a la Eurocámara: comicios de segundo orden y en clave nacional

Las elecciones europeas no despiertan interés por sí mismas sino que son una ocasión de los partidos para marcar sus diferencias en torno a cuestiones clave de la política española. Un modo de afrontar la campaña que reproducen los medios analizados.

\section{REFERENCIAS BIBLIOGRÁFICAS}

ASOCIACIÓN DE EDITORES DE DIARIOS ESPAÑOLES (2010): Libro Blanco de la Prensa Diaria 2010, AEDE: Madrid.

BERGANZA, M. R. (2005): Periodismo Especializado e Información de la Unión Europea. Madrid: Ediciones Internacionales Universitarias.

-"Las elecciones al Parlamento Europeo como comicios de segundo orden. Estudio de la cobertura informativa en televisión (1999-2004)". Estudios sobre el Mensaje Periodístico, no 14 (2008a), pp. 15-31.

-"Medios de comunicación, 'espiral del cinismo' y desconfianza política. Estudio de caso de la cobertura mediática de los comicios electorales europeos". ZER, vol. 13, nº 25 (2008b), pp. 121-139.

CANEL, M. J. (2006): Comunicación política. Una guía para su estudio y práctica. Madrid: Tecnos.

CANEL, M. J. y BERGANZA, M. R.: La campagna in Spagna: localizzatione mediatica o discorso político europeo? En MARINI, R. (ed.) (2001): L'Europa dell'euro e della guerra. La campagna electtorale 1999 in Italia e in sette paesi dell'Unione. Roma: RAI, VQPT, pp. 91-212.

CANEL, M. J. e INNERARITY, C.: La campaña electoral en la televisión. En MARTíNEZ, A. y MÉNDEZ, M. (eds.) (2000): Las elecciones al Parlamento Europeo, 1999. Valencia: Tirant lo Blanch, pp. 171-190.

DAHL, R. (1999): La democracia. Una guía para los ciudadanos. Madrid: Taurus.

GARCíA, M. M. y PÉREZ, C. (2009): ¿La Europa de los ciudadanos? Las elecciones del 7 de junio de 2009 al Parlamento Europeo y su reflejo en los medios de comunicación impresos: El País y El Mundo. Comunicación presentada en el II Congreso de la Asociación Española de Investigación en Comunicación (AE-IC), Málaga.

DE VREESE, C. H.: "Second-Rate Election Campaigning? An Analysis of Campaign Styles in European Parliamentary Elections". Journal of Political Marketing, vol. 8, no 1 (2009), pp. 7-19.

DE VREESE, C. H.: Continuity and Change: The 2004 European Elections in the Netherlands. En MAIER M. y TENSCHER, J. (eds.) (2006): Campaigning for Europe. Parties, Campaigns, Mass Media and the European Parliament Elections 2004. Londres: LIT Publishers, pp. 207-218.

KEVIN, D. (2003): Europe in the Media. A Comparison of Reporting, Representation, and Rhetoric in National Media Systems in Europe. Mahwah: Lawrence Erlbaum Associates.

MAAREK, P. (dir.) (2007): Chronique d'un "non" annoncé: la communication politique et I'Europe (juin 2004-mai 2005). Paris: L'Harmattan. 
MAIER M. y TENSCHER, J. (eds.) (2006): Campaigning for Europe. Parties, Campaigns, Mass Media and the European Parliament Elections 2004. Londres: LIT Publishers.

MORATA, F.: El Parlamento Europeo: una institución en busca de su papel político. En MARTÍNEZ A. y MÉNDEZ M. (eds.) (2000): Las elecciones al Parlamento Europeo, 1999. Valencia: Tirant lo Blanch, pp. 13-59.

REIF, K.: The Second-order National Elections. En REIF, K. (ed.) (1985): Ten European Elections. Aldershot: Gorver, pp. 1-36.

RUIZ, A. M.: La identidad europea de los españoles: sentido pasado y presente de la identificación con Europa y España. En Real Instituto Elcano de Estudios Estratégicos e Internacionales (2004): Documento de Trabajo nº 61/2004 (en línea. Consulta: 6 de noviembre de 2010). Http://realinstitutoelcano.org/wps/portal/rielcano/contenido?WCM_ GLOBAL_CONTEXT=/elcano/elcano_es/zonas_es/dt61-2004

SÁNCHEZ ARANDA, J. J.: Análisis de contenido cuantitativo de medios. En BERGANZA, M. R. y RUIZ, J. A. (coord.) (2005): Investigar en comunicación. Guía práctica de métodos y técnicas de investigación social en comunicación. Madrid: McGraw Hill, pp. 207-228.

SOTELO, J.: "Las encrucijadas del periodista sobre asuntos comunitarios: periodismo, nacionalismo y europeísmo”. Ámbitos, nº 17 (2008), pp. 271-288.

STEINBRECHER, M. y HUBER, S.: European Elections' Turnout from 1979 to 2004. En MAIER M. y TENSCHER, J. (eds.) (2006): Campaigning for Europe. Parties, Campaigns, Mass Media and the European Parliament Elections 2004. Londres: LIT Publishers, pp. 15-30.

TENSCHER, J. y MAIER M.: "European Parliamentary Elections 2004. Studies on Political Campaigning and Media Framing of a Second-Order Event". Journal of Political Marketing, $\mathrm{n}^{\circ} 8$ (2009), pp. 1-6.

TIGASSON, K. L.: "Strategic Miscalculations. Election Campaigns to the European Parliament in Estonia 2004". Journal of Political Marketing, $\mathrm{n}^{\circ} 8$ (2009), pp. 46-58.

ZUGASTI, R.; GARCÍA, C.; LUMBIERRES, C.; VERÓN, J. J., y SABÉS, F. (2011): La campaña de las elecciones generales de 2008 en la prensa diaria. El caso aragonés. Zaragoza: Asociación de Periodistas de Aragón.

\section{Breve semblanza de las autoras}

Patricia Lafuente Pérez es licenciada en Periodismo y Doctora en Comunicación por la Universidad de Navarra. Su investigación y su docencia, que desarrolla en la actualidad en la Facultad de Comunicación de la Universidad San Jorge, están vinculadas a las áreas de la Sociología y de Comunicación Política. Pertenece al grupo de investigación "Medios de comunicación y campañas electorales en Aragón", reconocido como grupo emergente por el Gobierno de Aragón.

Cristina Zurutuza Muñoz es Doctora en Comunicación por la Universidad de Navarra. Ha sido research scholar en The George Washington University e análisis San Jorge, donde 
80 El tratamiento informativo de las elecciones de 2009 a la Eurocámara: comicios de segundo orden y en clave nacional

desarrolla su tarea docente e investigadora en el ámbito de la comunicación de crisis y la comunicación política. Es integrante del grupo de investigación "Medios de comunicación y campañas electorales en Aragón", reconocido como emergente por el Gobierno de Aragón.

(Recibido el 07-09-2011; aceptado el 07-11-2011) 